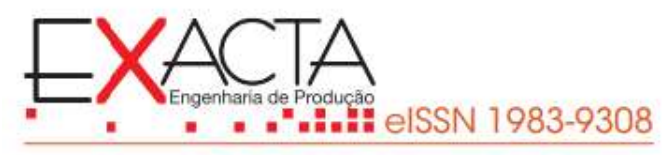

\title{
Mapeamento dos custos de logística reversa em uma indústria de embalagens
}

\author{
Mapping of reverse logistics costs in a packaging industry
}

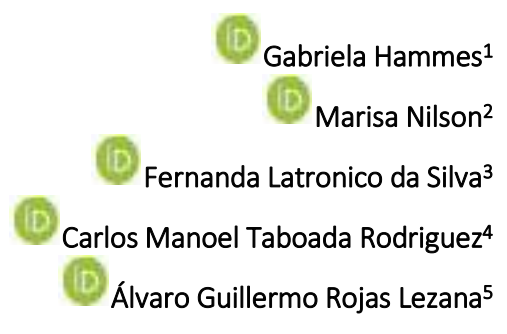

${ }^{1}$ Mestre em Engenharia de Produção, Universidade Federal de Santa Catarina. gabihammes15@gmail.com

${ }^{2}$ Doutoranda em Engenharia de Produção, Universidade Federal de Santa Catarina. isanilson80@gmail.com

\begin{abstract}
${ }^{3}$ Doutora em Engenharia de Produção, Universidade Federal de Santa Catarina.

fernanda.latronico@posgrad.ufsc.br
\end{abstract}

${ }^{4}$ Doutor em Engenharia Econômica, professor da Universidade Federal de Santa Catarina. carlos.taboada@ufsc.br

${ }^{5}$ Doutor em Ingeniería Industrial, professor da Universidade Federal de Santa Catarina. alvaro.lezana@ufsc.br

\begin{abstract}
Resumo
Com a questão da sustentabilidade em alta, as empresas estão começando a tomar medidas para atenuar os impactos ambientais gerados pelo processo produtivo. Além disso, a legislação está se tornando mais severa quanto às questões ambientais, e este fator envolve o descarte correto dos resíduos produzidos pelas empresas. A partir do conhecimento dos custos envolvidos na logística reversa, a empresa pode conhecer melhor este processo e implementar melhorias a fim de diminuir os desperdícios. O objetivo deste trabalho é mapear a logística reversa de uma empresa de embalagens industriais no norte de Santa Catarina. Os dados foram obtidos por meio de um estudo de caso. A partir do mapeamento da LR foi possível levantar os custos envolvidos nesta prática e distribuí-los para as atividades logísticas envolvidas. Após a análise destes custos, comprovou-se que a logística reversa pode gerar uma receita positiva para a empresa, além de originar ganhos ambientais e sociais.
\end{abstract}

Palavras-chave: Logística Reversa. Custos. Reciclagem.

\section{Abstract}

With the issue of sustainability on the rise, companies are beginning to take measures to mitigate the environmental impacts generated by the production process. In addition, legislation is becoming more severe on environmental issues, and this factor involves the correct disposal of waste produced by companies. From the knowledge of the costs involved in this reverse flow, the company can better understand this process and implement improvements in order to reduce waste. The objective of this work is to map the reverse logistics of an industrial packaging company in northern Santa Catarina, Brazil. The data were obtained by means of a case study. From the RL mapping it was possible to raise the costs involved in this practice and distribute them to the logistics activities involved. After analyzing these costs, it was verified that the reverse logistics can generate positive revenue for the company, besides generating environmental and social gains.

Keywords: Reverse Logistics. Costs. Recycling. 


\section{Introdução}

Entre os desafios das empresas contemporâneas cita-se a premência de se manter no mercado de forma competitiva. Neste sentido, a logística se apresenta como importante ferramenta. A Logística Empresarial é a gestão coordenada das atividades logísticas que busca melhorar o desempenho da empresa (Ballou, 2004). No Brasil, esta prática é caracterizada pela preocupação com os custos de estoques e transporte, pela competição interna e externa, pela agilidade nos processos, pela formação de especialistas e pela necessidade de melhorar a matriz de transportes (Leite, 2009).

Para garantir a permanência no mercado, as empresas reconhecem que além da busca pelo lucro é preciso atender aos interesses sociais, ambientais e governamentais (Leite, 2009). Além das preocupações com a logística direta inclui-se o movimento de retorno de bens na cadeia de suprimentos e as questões ambientais (Genchev, 2009).

Assim, o objetivo das empresas atualmente é buscar soluções para o excesso de resíduos gerados no decorrer da cadeia produtiva, e uma das formas de atingir este objetivo é por meio da logística reversa (LR). Além disso, a legislação está cada vez mais severa quanto ao descarte incorreto do lixo, fazendo com que as empresas repensem suas responsabilidades sobre seus produtos após seu uso (Daher, Silva, \& Fonseca, 2006).

Para Pokharel e Mutha (2009), a LR inclui desmontagem, remanufatura, reciclagem, além de planejamento da cadeia de suprimentos, coordenação, controle de estoques. Atualmente, entre os principais objetivos da LR estão: a gestão de resíduos, a recuperação de materiais por meio da reciclagem e da reutilização dos produtos. De acordo com Mendes et al. (2016), o produto ou resíduo precisa ser inspecionado e classificado para determinar a melhor forma de sua disposição.

No que se refere aos custos de uma forma geral, o acompanhamento é feito a partir de sistemas de custeio tradicionais, que são organizados de forma funcional, já a Logística possui uma abordagem processual, pois envolve recursos de vários departamentos, o que exige outras técnicas para o cálculo de seus custos (Rodríguez et al., 2014). Para se calculado o custos na LR devem ser calculados os custos de todas as atividades que envolvem a Logística, a saber: transporte, armazenagem, estoque, informações e administração.

Entretanto, poucos autores investigaram os custos na LR, como é o caso de Teunter, Lannb e Inderfurtha (2000) que criaram um modelo de logística reversa específico com custos de instalação, prazos de entrega e eliminação. Os autores afirmam que possíveis reduções de custos aumentou a atenção para a implantação da LR, além das questões legislativas e ambientais. Kannan (2009) propõe um modelo para avaliar e selecionar o melhor provedor de logística reversa de empresas terceirizadas. O autor alega que a LR tem como foco o fluxo de materiais do cliente para o fornecedor (ou diposição alternativa) com o objetivo de maximizar o valor do item devolvido ou minimizar o custo total. Ramos, 
Gomes e Povoa (2013) buscaram dar suporte para decisões táticas e de planejamento operacional de sistemas de logística reversa considerando os aspectos econômicos, ambientais e sociais.

Reconhecendo que há poucas pesquisas que abordam os custos na LR, este artigo tem como objetivo fazer um mapeamento dos custos de LR em uma empresa de embalagens industriais que atua no norte de Santa Catarina, Brasil. Os resultados demonstram que a implantação da LR pode gerar ganhos econômicos para empresa, além de adequar-se à Política Nacional dos Resíduos Sólidos (PNRS), Lei vigente no Brasil desde 2012.

$\mathrm{O}$ artigo foi organizado da seguinte forma. A seção 2 apresenta a revisão da literatura. Na seção 3 encontram-se os procedimentos metodológicos. Na seção 4 encontra-se o estudo de caso e na seção 5 a conclusão.

\section{Revisão da literatura \\ 2.1 Logística Reversa (LR)}

Os processos produtivos envolvem fornecedores, empresas e clientes, que estão entrelaçados pelos fluxos de informações, materiais e capitais. Alinhado ao valor do produto, está a carga ambiental e social suportadas no decorrer das diferentes fases de produção (Seuring \& Muller, 2008). Com o passar dos anos, os impactos ambientais e os resíduos gerados no decorrer da cadeia produtiva começaram a ser questionados.

No que se refere à Logística, um dos pontos relevantes são os canais de distribuição. Estes canais podem ser diretos (da empresa para os consumidores) ou reversos, levando os produtos de pós-venda ou de pós-consumo de volta para o ciclo produtivo (Leite, 2009). Para Silva et al. (2013), as empresas tem-se tornado cada vez mais proativas com foco em melhorar sua imagem corporativa por meio de mudanças que envolvem aspectos ambientais e sociais. Nesse sentido, a preocupação das empresas com o retorno dos resíduos fez com que os bens pós-venda e pós- consumo se tornasse parte integrante do processo produtivo.

Ravi e Shankar (2005b) afirmam que os gestores devem demonstrar compromisso da LR com outros objetivos organizacionais, integrando todos os membros da cadeia. De acordo com os mesmos autores, deve ser fornecido suporte contínuo para a LR nos planos estratégicos e nos planos de ação para o sucesso da implementação.

Entretanto, a LR pode ser um processo de geração de custos devido à falta de planejamento inverso, uma vez que é um processo mais complexo e envolve outros fatores para a implementação (Baenas et al., 2011). Contudo, com o planejamento apropriado pode ser uma prática lucrativa e ambientalmente benéfica.

Como a LR é uma área que não envolve lucros, apenas custos, muitas empresas deixam de praticá-la (Daher, Silva, \& Fonseca, 2006). No entanto, segundo Daga (2003, p.3), “um sistema eficiente de LR pode vir a transformar um processo de retorno altamente custoso e complexo em uma 
vantagem competitiva". Muitas empresas afirmam que a LR é a última fronteira em redução de custos.

Segundo Barker e Zabinsky (2010), existem três motivos para que as empresas invistam em LR. O primeiro é o atendimento a legislação, presente em diversos países, quanto a remanufatura, reutilização e reciclagem dos materiais. O segundo está na existência, em muitos casos, de um significativo valor econômico nos produtos de pós-consumo. A terceira motivação está na imagem da empresa, pois os consumidores buscam por empresas que adotem uma solução "verde" para seus resíduos.

\subsection{Custos na logística reversa}

De acordo com Min e Ko (2006), a crescente preocupação com os custos de retornos de produtos e a redução das margens de lucro, tornou a LR um diferencial competitivo, uma vez que a empresa pode economizar uma quantidade substancial de custos de transporte, estoque e armazenagem associados aos retornos de produtos.

Para o cálculo dos custos da LR é necessário que se calcule os custos de cada atividade logística separadamente.

\subsubsection{Custos de Transporte}

O cálculo dos custos de transporte varia de acordo com a frota própria ou terceirizada. Quando a frota é terceirizada basta analisar o montante pago por mês para a transportadora. Mas quando o transporte é próprio é necessário que se determine os custos desta atividade, que podem ser divido em fixos e variáveis (Rodríguez et al., 2014).

\subsubsection{Custos de Estoque}

Os estoques estão ligados a dinheiro imobilizado. Para Rodríguez et al. (2014), os custos de estoques envolvem: custo de aquisição, custo de manutenção, custo de estoque em trânsito e custo da falta de estoque.

O custo de aquisição engloba o preço ou o custo de fabricação do produto (Ballou, 2004). O custo de manutenção de estoque está relacionado ao risco, as perdas, aos seguros e ás avarias decorrentes da manutenção e custódia dos estoques (Rodríguez et al., 2014). O custo de estoque em transito envolve as perdas que podem ocorrer durante o transito do produto e o capital imobilizado durante o trânsito (Rodríguez et al., 2014). O custo da falta de estoque se refere às vendas perdidas e aos pedidos atrasados (Ballou, 2004). 


\subsubsection{Custos com Armazenagem, manuseio de materiais e embalagens}

Rodríguez (2014) lista os seguintes custos envolvidos no armazém:

- Custo de capital referente à construção, compra ou aluguel do armazém;

- Manutenção, água, eletricidade, impostos e seguro;

- Equipamentos de movimentação e armazenagem de materiais: aluguel ou manutenção, depreciação e custo de capital dos equipamentos;

- Mão de obra e encargos;

- Tecnologia da informação e comunicação: aluguel ou manutenção, depreciação e custo de capital das tecnologias.

\section{Procedimentos metodologicos}

\subsection{Etapas da pesquisa}

O primeiro passo foi estabelecer uma base teórica com material que abordasse os conceitos necessários para subsidiar a pesquisa. Para tanto, foram feitas buscas de palavras-chave, primeiramente divididas em dois eixos:

Eixo 1: "reverse logistic" OR "reverse logistics".

Eixo 2: "cost" OR "costs" OR "charge" OR "expenses" OR "expense" OR "expenditure" OR "spend" OR "outlay" OR "disbursing" OR "disburse" OR "outgo".

As palavras-chave foram pesquisadas nas seguintes base de dados: Scopus, Science Citation Index Expanded (Web of Science), OneFile (GALE) e ProQuest. A busca foi limitada para artigos científicos e de revisão, publicados a partir de 2005. Foram encontrados um total de 1396 artigos.

Primeiramente foram excluídos os artigos repetidos e em seguida iniciou-se o processo de seleção. A primeira seleção foi feita a partir da leitura dos títulos dos artigos. A segunda seleção ocorreu a partir da leitura dos resumos, selecionando apenas os que se encaixam com o tema da pesquisa. A terceira seleção ocorreu a partir da leitura integral dos artigos. Essa etapa pode visualizada na Figura 1 a seguir. 
Figura 1 - seleção dos artigos

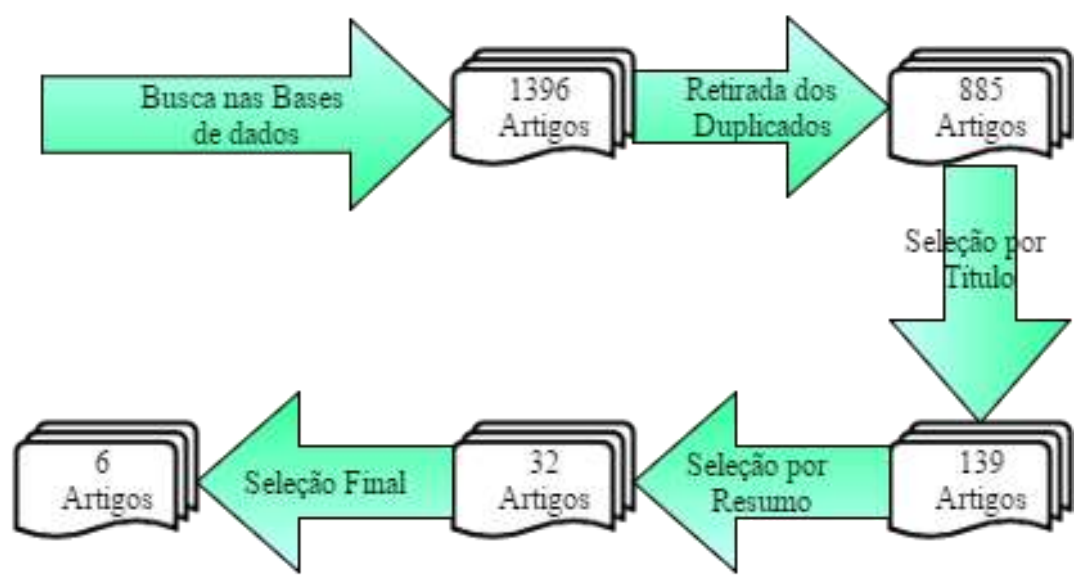

Fonte: Os autores.

Grande parte dos artigos encontrados não se identificou com o tema da pesquisa, pois não tratavam dos custos da LR como um todo. Alguns artigos destinam-se somente aos custos de transporte, outros nem abordavam a temática custos, apenas descrevem a LR e relatam casos da sua aplicação. Em meio à leitura dos artigos foram encontrados autores que se destacaram a partir de suas colocações sobre o assunto. Assim, a partir do referencial teórico destas obras, foram encontradas outras publicações pertinentes ao assunto e utilizadas na construção do referencial teórico desta pesquisa.

Na segunda etapa, foi realizado um único estudo de caso longitudinal, o que permite um maior aprofundamento na coleta de dados (Cauchick Miguel \& Sousa, 2012). O local de aplicação prática desse estudo ocorreu em uma indústria de embalagens industriais que atua em todo o Brasil.

A coleta de dados aconteceu por meio de uma visita a empresa e de uma entrevista com o gerente. Os dados desta entrevista permitiram a elaboração do mapeamento da LR da empresa e a análise dos custos envolvidos nesta prática.

\section{Estudo de caso}

O presente estudo tem como objetivo analisar os custos envolvidos no sistema de LR de uma indústria transformadora de polímero. Por questão de sigilo o nome da empresa escolhida para o estudo de caso não é divulgado.

\subsection{A empresa}

A empresa objeto de estudo ocupa posição de destaque como produtora de embalagens para produtos industriais e atua em inúmeras áreas. Sua matriz encontra-se no estado de Santa Catarina e 
conta com diversas unidades no território brasileiro. Atua no mercado há várias décadas e em 2007 iniciou suas operações em LR e reciclagem.

Com o surgimento da Política Nacional dos Resíduos Sólidos (PNRS), a empresa viu a necessidade de dar um destino correto para os resíduos gerados e, após alguns estudos, criou-se uma Central de Reciclagem (CR).

A CR é responsável pela reciclagem do produto de pós-consumo. Após a reciclagem cerca de $20 \%$ do material retorna para as instalações da empresa para ser reprocessado e novamente inserido na cadeia produtiva como matéria-prima (MP). Os $80 \%$ restantes são vendidos para outras indústrias de transformação que também o utilizam como MP. A ampliação da LR faz parte da estratégia de crescimento sustentável da empresa. Em 2014 o programa de reciclagem foi ampliado para todas as unidades. Ocorreu também um aumento na divulgação do mesmo com a criação de um portal online que indica os pontos de coleta do material. Neste mesmo ano houve um aumento de $7 \%$ no número de parceiros envolvidos no recolhimento dos produtos de pós-consumo, o que corresponde a um total de 710 agentes.

\subsubsection{Mapeamento da LR}

Cada unidade da empresa possui uma CR. O fluxo reverso abrange um raio de 200 quilômetros de cada unidade. Esta abrangência pode ser maior dependendo da sua viabilidade.

A empresa possui parcerias com 3 tipos de fornecedores de sucata para a reciclagem, conforme Tabela 1 a seguir.

Tabela 1 - Fornecedores de Sucata

\begin{tabular}{l|l}
\hline Tipos de Fornecedores de Sucata & $\begin{array}{l}\text { Atividades realizadas } \\
\text { Cooperativas de Reciclagem }\end{array}$ \\
\hline Gestores de Resíduos & $\begin{array}{l}\text { Eesíduos, onde ocorre a sua reciclagem ou venda. } \\
\text { encaminham para aterros sanitários, centros ou }\end{array}$ \\
& $\begin{array}{l}\text { cooperativas de reciclagem, lixões, entre outros. Um dos } \\
\text { meios para a destinação dos resíduos é a empresa em } \\
\text { estudo. }\end{array}$ \\
\hline Empresas de Geração Direta & $\begin{array}{l}\text { Empresas que tem alta produção de resíduos e, para } \\
\text { atender a PNRS, encaminham os resíduos para a CR a fim } \\
\text { de que tenham uma destinação correta por meio da } \\
\text { reciclagem. }\end{array}$ \\
\end{tabular}

Fonte: Os autores. 
Nem sempre ocorre a participação dos 3 tipos de fornecedores em todas as unidades de reciclagem, algumas podem receber materiais somente de 1 ou de 2 tipos de fornecedores em determinado mês.

A partir destes fornecedores os resíduos são encaminhados para a CR. O transporte acontece de 4 formas distintas, conforme apresenta a Tabela 2.

Tabela 2 - Tipos de Transporte

\begin{tabular}{l|l}
\hline Transporte & Como é realizado \\
\hline Veículos próprios & $\begin{array}{l}\text { Utilizam o mesmo canal de distribuição da logística direta } \\
\text { da empresa para o fluxo reverso. }\end{array}$ \\
\hline Fretes reversos & $\begin{array}{l}\text { Caminhoneiros autônomos que procuram a empresa para } \\
\text { fazer um frete reverso. Cobram cerca de 40\% do valor do } \\
\text { frete normal. }\end{array}$ \\
\hline Veículos do fornecedor & $\begin{array}{l}\text { Quando o fornecedor é responsável pelo transporte. } \\
\text { Terceirizados }\end{array}$ \\
& $\begin{array}{l}\text { sucata quando o fornecedor possui uma carga completa e } \\
\text { nenhuma das outras opções de transporte está disponível. }\end{array}$ \\
\hline
\end{tabular}

Fonte: Os autores.

Ao chegar na CR os resíduos são descarregados em uma área destinada para o estoque de matéria-prima. Está área não possui nenhum tipo de controle de estoques. A partir deste estoque o material passa pela linha de produção onde é reciclado e, ao final, é embalado e armazenado. Cerca de $20 \%$ do material reciclado retorna para a linha de produção de embalagens industriais, que é a atividade principal da empresa. O restante é vendido para outras empresas como MP para diversos outros produtos.

Após a produção e venda de embalagens industriais, o produto chega ao cliente final e é consumido. Depois de ser consumido o material retorna para o ciclo por meio de um dos 3 tipos de fornecedores de resíduos, fechando o ciclo da LR. Na Figura 2 é possível visualizar ciclo da LR. 
Figura 2 - Gráfico de fluxo de processos da LR

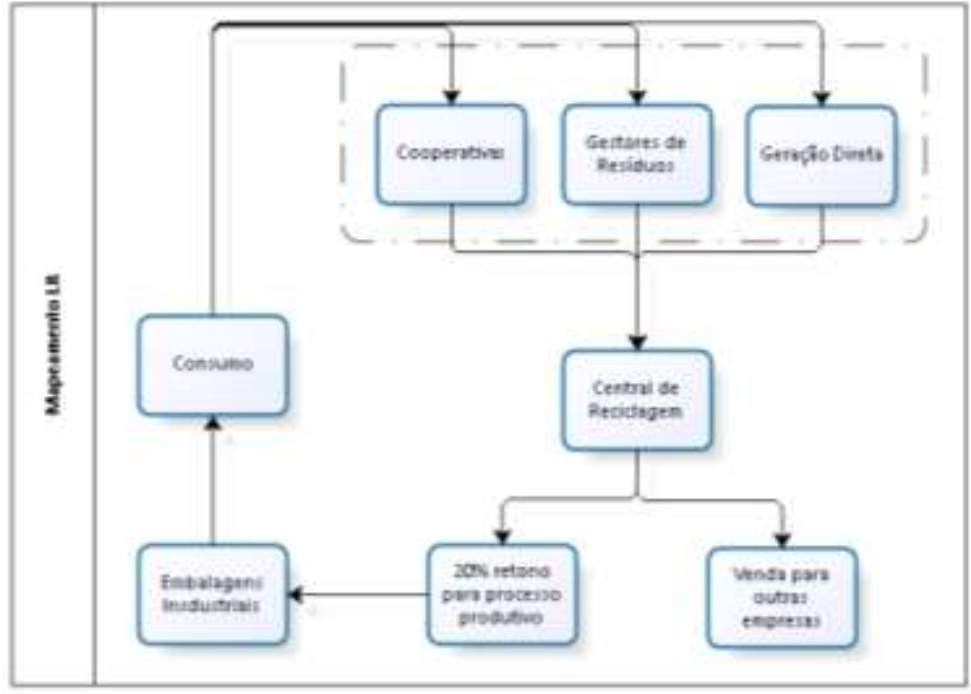

Fonte: Os autores.

Assim como a PNRS, o ciclo fechado da LR necessita de uma gestão compartilhada dos resíduos, tanto por parte da empresa como por parte dos consumidores, ao separar e reciclar seu lixo, e por parte dos gestores de resíduos, ao enviar estes para a reciclagem.

\subsection{Distribuição dos custos por atividades}

A empresa não possui um controle de custos detalhado da $L R$, somente os custos com transporte e aquisição de materiais são tratados com acuracidade. O restante dos custos foram mensurados pelos pesquisadores. A tabela 3 a seguir apresenta os dados do mês de setembro de 2016 para 6 unidades de reciclagem. A disponibilidade de dados é insuficiente para a utilização do Custeio de Vida Total, devido à falta de informações quanto aos custos de fabricação do produto e da logística direta.

Tabela 3 - Controle Financeiro Setembro-2016

\begin{tabular}{c|c|c|c|c|c|c|c}
\hline Uni. & $\begin{array}{c}\text { Cooperativas } \\
(\mathrm{kg})\end{array}$ & $\begin{array}{c}\text { Gestores } \\
(\mathrm{kg})\end{array}$ & $\begin{array}{c}\text { G. Direta } \\
(\mathrm{kg})\end{array}$ & Total $(\mathrm{Kg})$ & Frete & Sucata & Faturamento \\
\hline $\mathrm{A}$ & 6759 & 1655 & 40654 & 49068 & $\mathrm{R} \$ 11.590,00$ & $\mathrm{R} \$ 23.828,48$ & $\mathrm{R} \$ 372.159,06$ \\
\hline $\mathrm{B}$ & 3185 & 6020 & 6970 & 16175 & $\mathrm{R} \$ 5.539,90$ & $\mathrm{R} \$ 5.843,20$ & - \\
\hline $\mathrm{C}$ & 11665 & 6577 & 28763 & 47005 & $\mathrm{R} \$ 14.800,00$ & $\mathrm{R} \$ 26.062,00$ & $\mathrm{R} \$ 209.263,98$ \\
\hline $\mathrm{D}$ & 2579 & 600 & 17451 & 20630 & $\mathrm{R} \$ 550,00$ & $\mathrm{R} \$ 3.934,90$ & - \\
\hline E & - & - & 10186 & 10186 & $\mathrm{R} \$ 8.000,00$ & $\mathrm{R} \$ 907,00$ & - \\
\hline F & - & 570 & 3150 & 3720 & $\mathrm{R} \$ 95,52$ & $\mathrm{R} \$ 2.265,00$ & - \\
\hline TOTAL & 24188 & 15422 & 107174 & 146784 & $\mathrm{R} \$ 40.575,42$ & $\mathrm{R} \$ 62.840,58$ & $\mathrm{R} \$ 581.423,04$ \\
\hline
\end{tabular}

Fonte: Os autores. 
A unidade E não recebe sucada de cooperativas nem de gestores de resíduos, recebe apenas de empresas com geração direta. A unidade F não recebe material de cooperativas. As demais unidades recebem sucata dos 3 tipos de fornecedores.

\subsubsection{Custo de Transporte e Aquisição}

Os custos com transporte são variáveis pois dependem da forma como este transporte foi feito: por meio de veículos próprios da empresa ou de forma terceirizada. De qualquer maneira, os fretes sempre são feitos com carga fechada de $600 \mathrm{~kg}$.

Ao somar o total de sucata e dividir este valor por $600 \mathrm{~kg}$ resulta-se no número de fretes realizados no referido mês. É possível estimar o valor médio por frete e o custo de transporte para cada quilo de sucata transportado a partir do custo total com fretes disponibilizado. Estes valores foram calculados para as 6 CR's e podem ser observados no Quadro 2.

O custo de aquisição do quilo de sucata é resultado da divisão do valor total gasto com sucata pela quantidade total de quilos adquirida. Este valor encontra-se na Tabela 4, que também mostra o valor médio gasto com aquisição e transporte para cada unidade.

Tabela 4 - Custo de Transporte e Aquisição

\begin{tabular}{|c|c|c|c|c|c|c|c|c|}
\hline \multirow[b]{2}{*}{ Unidade } & \multirow[b]{2}{*}{$\begin{array}{l}\text { Total } \\
(\mathrm{Kg})\end{array}$} & \multicolumn{4}{|c|}{ TRANSPORTE } & \multicolumn{2}{|c|}{ AQUISIÇÃO } & \multirow[b]{2}{*}{$\begin{array}{l}\text { Custo } \\
\text { Médio }\end{array}$} \\
\hline & & $\begin{array}{c}\text { Custo Total } \\
\text { Frete }\end{array}$ & $\begin{array}{l}\text { № de } \\
\text { Fretes }\end{array}$ & $\begin{array}{c}\text { Custo } \\
\text { Médio por } \\
\text { Frete }\end{array}$ & $\begin{array}{c}\text { Custo } \\
\text { Médio por } \\
\text { Quilo }\end{array}$ & $\begin{array}{c}\text { Custo Total } \\
\text { Sucata }\end{array}$ & $\begin{array}{c}\text { Custo } \\
\text { Médio por } \\
\text { Quilo }\end{array}$ & \\
\hline$A$ & 49068 & $\mathrm{R} \$ 11.590,00$ & 82 & $\mathrm{R} \$ 141,72$ & $R \$ 0,24$ & $\mathrm{R} \$ 23.828,48$ & $R \$ 0,49$ & $\mathrm{R} \$ 0,72$ \\
\hline B & 16175 & $\mathrm{R} \$ 5.539,90$ & 27 & $R \$ 205,50$ & $R \$ 0,34$ & $\mathrm{R} \$ 5.843,20$ & $R \$ 0,36$ & $R \$ 0,70$ \\
\hline C & 47005 & $\mathrm{R} \$ 14.800,00$ & 78 & $\mathrm{R} \$ 188,92$ & $R \$ 0,31$ & $R \$ 26.062,00$ & $\mathrm{R} \$ 0,55$ & $\mathrm{R} \$ 0,87$ \\
\hline $\mathrm{D}$ & 20630 & $\mathrm{R} \$ 550,00$ & 34 & $\mathrm{R} \$ 16,00$ & $\mathrm{R} \$ 0,03$ & $\mathrm{R} \$ 3.934,90$ & $\mathrm{R} \$ 0,19$ & $\mathrm{R} \$ 0,22$ \\
\hline$E$ & 10186 & $\mathrm{R} \$ 8.000,00$ & 17 & $\mathrm{R} \$ 471,24$ & $\mathrm{R} \$ 0,79$ & $\mathrm{R} \$ 907,00$ & $\mathrm{R} \$ 0,09$ & $R \$ 0,87$ \\
\hline$F$ & 3720 & $\mathrm{R} \$ 95,52$ & 6 & $\mathrm{R} \$ 15,41$ & $\mathrm{R} \$ 0,03$ & $R \$ 2.265,00$ & $\mathrm{R} \$ 0,61$ & $\mathrm{R} \$ 0,63$ \\
\hline TOTAL & 146784 & $\mathrm{R} \$ 40.575,42$ & 245 & $\mathrm{R} \$ 173,13$ & $R \$ 0,29$ & $R \$ 62.840,58$ & $R \$ 0,38$ & $R \$ 0,67$ \\
\hline
\end{tabular}

Fonte: Os autores.

unidade $\mathrm{E}$ possui o menor custo de aquisição, apenas $\mathrm{R} \$$ 0,09 por quilo. Isso ocorre pois seus fornecedores são empresas que geram resíduos e os vendem para a unidade a um preço menor como forma de atender a PNRS. Apesar disso, o custo com transporte é o mais alto, elevando o seu custo médio. As unidades $\mathrm{D}$ e $\mathrm{F}$ possuem o menor custo de transporte, o que contribui para que a unidade $\mathrm{D}$ obtenha o menor custo médio. 


\subsubsection{Custo de Estoque}

A partir deste custo médio é possível calcular o custo do estoque. O custo de estoque leva em conta o custo de transporte pois, enquanto é transportado, o material esta imobilizado, e, legalmente, é considerado como estoque.

Os níveis de estoque por unidade são variáveis, pois estão relacionados com o consumo de embalagens industriais e da taxa de recolhimento de pós-consumo deste material. Atualmente o setor passa por uma retração, o que diminui o consumo das embalagens e, consequentemente, da sua reciclagem. Assim, o estoque médio será considerado como o total de estoque gerado no mês de setembro.

A taxa de imobilização de material praticada pela empresa é de 10\% ao ano. Como o custo será calculado por mês, é necessário transformar esta taxa para um valor mensal. Essa transformação é feita a partir da Equação 1, proposta por Casarotto Filho e Kopittke (2010).

$$
i_{a m}=-1+\left(1+i_{a a}\right)^{1 / 12}(\text { Eq. } 1)
$$

Onde:

$i_{a m}=$ taxa de imobilização de capital ao mês

$i_{a a}=$ taxa de imobilização de capital ao ano

$$
\begin{gathered}
i_{a m}=-1+(1+0,1)^{1 / 12} \\
i_{a m}=0,8 \%
\end{gathered}
$$

Assim, o custo de estoque para cada CR, foi calculado a partir da Equação 1 e pode ser observado na Tabela 5 a seguir.

Tabela 5 - Custo de Estoque

\begin{tabular}{c|c|c|c|c}
\hline & & & \\
\hline Unidade & Total (Kg) & Custo Médio & $\boldsymbol{i}_{a m}$ & Custo de Estoque \\
\hline A & 49068 & $\mathrm{R} \$ 0,72$ & 0,0080 & $\mathrm{R} \$ 282,43$ \\
\hline $\mathrm{B}$ & 16175 & $\mathrm{R} \$ 0,70$ & 0,0080 & $\mathrm{R} \$ 90,77$ \\
\hline $\mathrm{C}$ & 47005 & $\mathrm{R} \$ 0,87$ & 0,0080 & $\mathrm{R} \$ 325,84$ \\
\hline $\mathrm{D}$ & 20630 & $\mathrm{R} \$ 0,22$ & 0,0080 & $\mathrm{R} \$ 35,76$ \\
\hline $\mathrm{E}$ & 10186 & $\mathrm{R} \$ 0,87$ & 0,0080 & $\mathrm{R} \$ 71,03$ \\
\hline $\mathrm{F}$ & 3720 & $\mathrm{R} \$ 0,63$ & 0,0080 & $\mathrm{R} \$ 18,82$ \\
\hline TOTAL & 146784 & $\mathrm{R} \$ 0,67$ & 0,0080 & $\mathrm{R} \$ 784,47$ \\
\hline
\end{tabular}

Fonte: Os autores. 


\subsubsection{Central de Reciclagem A}

A CR A é a unidade de reciclagem da matriz da empresa, em Santa Catarina. Foi a primeira CR a ser criada, em 2007, e está mais organizada e desenvolvida em comparação com as demais. Esta CR conta com 21 funcionários, 3 no setor administrativo e 18 na área de reciclagem e manuseio de materiais. No mês de setembro de 2016, a CR A atingiu um faturamento de R\$372.159,06.

Os custos com transporte, aquisição de sucata e estoque já foram calculados anteriormente, restando o cálculo de armazenagem, manuseio de materiais e embalagem.

\subsubsection{Custos com armazenagem, manuseio e embalagem}

A empresa possui um galpão próprio destinado somente aos processos de LR. Neste galpão ocorre o processo de reciclagem e armazenamento dos materiais, além de um escritório para o setor administrativo.O custo de capital referente a construção e pode ser estimado por meio da depreciação do galpão. Imóveis são depreciados, geralmente, em 25 anos, isso resulta em uma taxa de $4 \%$ ao ano. A partir da Equação 1, a taxa de depreciação mensal é igual a 0,33\%. Esta taxa deve ser aplicada sobre o valor do imóvel. O galpão possui uma área construida de aproximadamente $2.250 \mathrm{~m}^{2}$, o valor por metro quadrado de um galpão em alvenaria, determinado pela prefeitura local no bairro onde a empresa se encontra é de $\mathrm{R} \$ 169,95$. Assim, estima-se que o galpão possua o valor de $\mathrm{R} \$$ 382.387,50. Aplicada a taxa, a depreciação mensal é de $\mathrm{R} \$ 1.251,84$.

A empresa possui um gasto médio anual de $\mathrm{R} \$ 20.000,00$ com impostos. Ao dividir este valor por 12 meses resulta-se em um custo médio mensal de R\$ 1.666,67.

Tabela 6 - Custo de Armazenagem

\begin{tabular}{l|lc}
\hline Armazenagem & $\mathrm{R} \$$ & $10.752,00$ \\
\hline Salários e Encargos Administrativo & $\mathrm{R} \$$ & $32.256,00$ \\
\hline Processos de Armazém & & \\
\hline Manutenção & $\mathrm{R} \$$ & $20.000,00$ \\
\hline Energia & $\mathrm{R} \$$ & 200,00 \\
\hline Água & $\mathrm{R} \$$ & $1.666,67$ \\
\hline Impostos & $\mathrm{R} \$$ & $1.251,84$ \\
\hline Custo de Capital & $\mathrm{R} \$$ & $66.126,50$ \\
\hline TOTAL & & \\
\hline
\end{tabular}

Fonte: Os autores.

A empresa não possui equipamentos de movimentação, embalagens ou tecnologia da informação envolvidos nos processos de LR. 


\subsection{Análise dos custos}

Após definir os custos de cada atividade logística, pode-se fazer uma análise dos mesmos. O Quadro 2 mostra uma divergência nos custos de transporte e aquisição para cada unidade. A variação no custo de aquisição, que é de $R \$ 0,09$ a $R \$ 0,61$, ocorre devido aos 3 tipos de fornecedores, onde cada um, em cada cidade, pratica um preço diferente. Já para o transporte a variação fica entre $\mathrm{R} \$$ 0,03 e R\$ 0,78. Esta oscilação acontece devido a forma de transporte praticada e a distância entre a CR e seus fornecedores.

Os custos da CR A estão resumidos na Tabela 7. Este quadro também mostra a porcentagem de participação nos custos de cada atividade. Nota-se que os custos com armazenagem possuem a maior contribuição. Isso se deve ao fato de estarem inclusos nesta atividade todos os custos com a reciclagem do material e todas as pessoas envolvidas neste processo.

Tabela 7 -Custos Logísticos da CR A

\begin{tabular}{c|c|c}
\hline \multicolumn{3}{|c|}{ Custos Logísticos } \\
\hline Atividades & Custo & $\%$ \\
\hline Transporte & $\mathrm{R} \$ 11.590,00$ & $14,9 \%$ \\
\hline Estoque & $\mathrm{R} \$ 282,43$ & $0,4 \%$ \\
\hline Armazenagem & $\mathrm{R} \$ 66.126,50$ & $84,8 \%$ \\
\hline TOTAL & $\mathrm{R} \$ 77.998,94$ & $100,0 \%$ \\
\hline
\end{tabular}

Fonte: Os autores.

A partir destes custos é possível analisar o impacto dos custos logísticos no faturamento da CR A. Basta dividir os custos pelo faturamento da unidade no referido mês, que foi de $\mathrm{R} \$ 372.159,06$. Os custos logísticos consomem 20,96\% do faturamento, ou seja, a cada real faturado $\mathrm{R} \$ 0,21$ são gastos em logística. Este faturamento é proveniente da venda do material reciclado para outras empresas transformadoras de polímero.

O valor gasto com estoque é muito pequeno, somando menos de 1 centavo a cada real faturado. Já os custos com transporte e armazenagem são mais expressivos, somando $R \$ 0,03$ e $\mathrm{R} \$ 0,18$ respectivamente. 
Tabela 8 - Custos logísticos por real faturado

\begin{tabular}{c|cc}
\hline \multicolumn{3}{|c}{ Custos Logísticos/Faturamento } \\
\hline Atividades & Valor (centavos por real faturado) \\
\hline Transporte & $\mathrm{R} \$$ & 0,031 \\
\hline Estoque & $\mathrm{R} \$$ & 0,001 \\
\hline Armazenagem & $\mathrm{R} \$$ & 0,178 \\
\hline TOTAL & $\mathrm{R} \$$ & 20,96 \\
\hline
\end{tabular}

Fonte: Os autores.

Os valores indicados no Quadro 6 mostram a dimensão do impacto da logística na competitividade da empresa. A partir desta tabela é possível saber qual a atividade merece maior atenção na busca pela redução de custos e aumento dos lucros.

Como a CR A é composta apenas por processos de LR, todos os custos da unidade são custos logísticos e já foram calculados. Assim, pode-se obter o lucro bruto do mês de setembro de 2016 para a CR A, ao diminuir os custos logísticos e os custos com aquisição da sucata do faturamento. O lucro bruto pode ser visto na Tabela 9 a seguir.

Tabela 9 - Lucro Bruto da CR A

\begin{tabular}{l|l}
\hline \multicolumn{2}{c}{ Central de Reciclagem A } \\
\hline Faturamento & $\mathrm{R} \$ 372.159,06$ \\
\hline Custos & $\mathrm{R} \$ 77.998,94$ \\
\hline Custo de aquisição & $\mathrm{R} \$ 23.828,48$ \\
\hline Lucro Líquido & $\mathrm{R} \$ 270.331,64$
\end{tabular}

Fonte: Os autores.

Assim, a empresa em estudo mostra que é possível não somente reduzir custos, mas também obter resultados positivos ao implementar a LR na empresa.

\subsection{Discussão}

Apesar de a empresa obter uma receita positiva com os processos de LR, alguns pontos podem ser melhorados para que este resultado seja mais satisfatório. Um dos pontos seria um melhor controle de seus estoques. Ainda que o impacto dos custos de estoque não seja tão significativo, uma melhoria no recebimento e organização dos materiais poderia reduzir gastos com mão de obra e tempo em estoque. Ao reduzir gastos com mão de obra, consequentemente se reduz gastos de armazenagem, que é a atividade com maior peso nos custos logísticos. 
De acordo com Min e Ko (2006), com implantação da LR a empresa pode economizar uma quantidade significativa de custos de transporte, estoque e armazenagem associados aos retornos de produtos. A empresa possui um galpão próprio destinado somente aos processos de $L R$, o que facilita o processo. Neste galpão ocorre o processo de reciclagem e armazenamento dos materiais, além de um escritório para o setor administrativo.

Conforme Ko e Evans (2007), uma das principais dificuldades associadas à implementação de atividades de logística reversa é o grau de incerteza em termos de tempo e quantidade de produtos. Assim, a gestão do fluxo de retorno geralmente requer uma infraestrutura especializada e um custo e tempo de manuseio relativamente altos.

A implementação da $L R$, especialmente nos retornos de produtos, permite economias no custo de estoques, custo de transporte e custo de descarte de resíduos (Rogers \& Tibben-Lembke, 1999). Entretanto, a empresa não possui um controle de custos detalhado da LR, somente os custos com transporte e aquisição de materiais são tratados em detalhe. Além disso, não possui equipamentos de movimentação ou tecnologia de informação envolvida nos processos de LR.

De acordo com Ko e Evans (2007), a implementação da LR exige uma infraestrutura especializada que necessite de sistemas especiais de informação para rastreamento, captura de equipamentos para processamento de retornos, e processos de fabricação padronizados especializados em toda a cadeia (Ko \& Evans, 2007). Com os crescentes custos de retorno de produtos e margens de lucro decrescentes, a manipulação ótima dos retornos do produto pode ser um diferencial competitivo (Min \& Ko, 2008).

Além disso, a disponibilidade de dados é insuficiente para a utilização do Custeio de Vida Total, devido à falta de informações quanto aos custos de fabricação do produto e da logística direta. De acordo com Daher et al. (2006), a inclusão de um sistema de LR necessita da abordagem do custeio do ciclo de vida total, pois as empresas passam a ser responsável por seus produtos até o final da sua vida útil. Nesse sentido, para adotar a LR é necessário estender o ciclo de vida dos materiais e planejar de forma eficiente o retorno dos produtos ao ciclo logístico (Guarnieri et al., 2015).

De uma forma geral, no que se referem à redução dos custos, as iniciativas relacionadas às práticas de LR têm trazido retornos significativos para a empresa. Assim, comprova-se que é possível reduzir custos e obter resultados positivos ao implementar a LR. Ravi e Shankar (2005b) afirmam que deve ser fornecido suporte contínuo para a LR nos planos estratégicos e nos planos de ação para o sucesso da implementação.

\section{Conclusões}

O objetivo deste trabalho foi analisar os custos da LR de bens pós-consumo em uma indústria transformadora de polímero. O objetivo foi atingido ao revelar o impacto de cada atividade logística 
no faturamento da empresa, e assim, permitir, por parte dos gestores, uma visão mais ampla do processo e auxiliar na tomada de decisões quanto ao fluxo reverso.

Primeiramente, foi realizado o mapeamento das atividades logísticas envolvidas no processo. O mapeamento contribuiu para levantar os custos de cada atividade voltada para a LR. A partir de alguns dados disponibilizados pela empresa, foi possível calcular os custos de cada atividade e a sua influência nos custos logísticos, além de mostrar o valor gasto pela empresa com a LR para cada real faturado. Este levantamento revelou qual a atividade tem maior contribuição nos custos do fluxo reverso.

Conclui-se com este estudo que as práticas de LR diminuem os custos da empresa e geram uma receita positiva, além de ganhos ambientais e sociais.

O conhecimento do processo da LR, por meio do mapeamento e dos custos envolvidos em cada atividade pode ajudar a empresa a melhorar algumas práticas específicas. Embora a LR da empresa esteja organizada e obtém um resultado positivo no fim do mês, a busca por melhorias no processo é contínua.

Entretanto, algumas limitações foram encontradas durante o andamento da pesquisa. Poucas empresas possuem um sistema de LR implantado e controlado. As empresas que dispõem deste sistema têm receio em disponibilizar seus dados financeiros. Assim, a principal limitação foi a obtenção dos dados para a pesquisa. Outro fator foi a distância das atividades em relação à empresa, que dificultou uma maior interação com o processo.

Como sugestão para trabalhos futuros, pode-se realizar uma análise comparativa dos custos da logística direta com a LR, levantando os dados de toda a cadeia logística da empresa. Outra sugestão é a criação de um modelo para o cálculo dos custos da LR, que serviria como base para as empresas que ainda não possuem o fluxo reverso implantado, ou, se já possuem, para que possam aprimorá-lo. Também é interessante a criação de um mapeamento da LR no Brasil, mostrando como esse sistema é utilizado no país e quais as maiores dificuldades na sua implantação.

\section{Referências}

Albino, V., Balice, A., \& Dangelico, R. M. (2009) Environmental strategies and green product development: an overview on sustainability-driven companies. Business Strategy and the Environment, 18(2), 83-96.

Ballou, R. H. (2004) Gerenciamento da cadeia de suprimentos /Logística empresarial. Tradução de Raul Rubenich. 5. ed. Porto Alegre: Bookman.

Barker, T. J., \& Zabinsky, Z. B. (2010) A solid reverse supply Chain is critical in an era of scarce resources. Industrial Engineer, 42(4), 38-44.

Barker, T. J., \& Zabinsky, Z. B. (2011) A multicriteria decision making model for reverse logistics using analytical hierarchy process. Omega, 39(5), 558-578. 
Bowersox, D. J., \& Closs, D. J. (2011) Logística empresarial: O processo de integração da cadeia de suprimento. São Paulo: Atlas.

Daga, A. (2003) Collaboration in reverse logistics. White Paper.

Daher, C. E., Silva, E. P. S., \& Fonseca, A. P. (2006) Logística Reversa: Oportunidade para Redução de Custos através do Gerenciamento da Cadeia Integrada de Valor. Brazilian Business Review, 3(1), 5873.

Genchev, S. E. (2009) Reverse logistics program design: A company study. Business Horizons, 52(2), 139-148.

Guarnieri, P., Sobreiro, V. A., Nagano, M. S., \& Serrano, A. L. M (2015) The challenge of selecting and evaluating third-party reverse logistics providers in a multicriteria perspective: a Brazilian case. Journal of Cleaner Production, 96(2), 209-219.

Kannan, G. (2009) Fuzzy approach for the selection of third party reverse logistics provider. Asia Pacific Journal of Marketing and Logistics, 21(3), 397-416.

Ko, H. J., \& Evans, G. W. (2007) A genetic algorithm-based heuristic for the dynamic integrated forward/reverse logistics network for 3PLs. Computers \& Operations Research, 34(1), 346-366.

Lacerda, L. (2002) Logística Reversa Uma visão sobre os conceitos básicos e as práticas operacionais. Rio de Janeiro: COPPEAD/UFRJ.

Leite, P. R. (2009) Logística reversa: meio ambiente e competitividade. 2 ed. São Paulo: Pearson Prentice Hall.

Leite, P. R. (2012) Custo ou oportunidade? Revista Tecnológica, 18(199), 44-50.

Mendes, G. S., Theis, V., Fagundes, C., Schereiber, D., \& Silva, M. O. (2016) Logística reversa: estudo de caso em uma indústria de artefatos plásticos. Exacta, 14(1), 37-45.

Min, H., Ko, H. J., \& Ko, C. S. (2006) A genetic algorithm approach to developing the multi-echelon reverse logistics network for product returns. Omega, 34(1), 56-69.

Min, H., \& Ko, H. J. (2008) The dynamic design of a reverse logistics network from the perspective of third-party logistics service providers. International Journal of Production Economics, 113(1), 176192.

Nunes, K. R. A., Mahler, C. F., \& Valle, R. A. (2009) Reverse logistics in the Brazilian construction industry. Journal of environmental management, 90 (12), 3717-3720.

Pokharel, S., \& Mutha, (2009) A. Perspectives in reverse logistics: A review. Resources, Conservation and Recycling, 53(4), 175-182.

Ramos, T. R. P., Gomes, M. I., \& Barbosa-Povoa, A. P. (2014) Planning a sustainable reverse logistics system: Balancing costs with environmental and social concerns. Omega, 48(1), 60-74.

Ravi, V., \& Shankar, V. (2005b) Analysis of interactions among the barriers of reverse logistics. Technological Forecasting \& Social Change, 72(8), 1011-1029.

Ravi, V., \& Shankar, V. (2005a) Analyzing alternatives in reverse logistics for end-of-life computers: ANP and balanced scorecard approach. Computers \& Industrial Engineering, 48(2), 327-356. 
Rodriguez, C. M. T., Follmann, N., Azevedo, J. M., Nilson, M., \& Cipullo, V. G. (2014) Custos Logísticos: Um Método para Apuração e Análise. Revista Mundo Logística, 42(1), 37-48.

Rodriguez, Carlos M. T. Apostila Tratamento de Custos Logísticos. Florianopolis: UFSC, 2016.

Rogers, D. S., \& Tibben-lembke, R. (1999) Going Backwards: Reverse Logistics Trends and Practices, RLEC Press, Pittsburgh, PA.

Rogers, D. S., \& TIbben-lembke, R. (2001) An examination of reverse logistics practices. Journal of Business Logistics, 22(2), 129-148.

Seuring, S., \& Muller, M. (2008) From a literature review to a conceptual framework for sustainable supply chain management. Journal of Cleaner Production, 16(15), 1699- 1710.

Silva, D. A. L., Renó, G. W. S., Sevegnani, G., Sevegnani, T. B., \& Truzzi, O. M. S.(2013) Comparison of disposable and returnable packaging: a case study of reverse logistics in Brazil. Journal of Cleaner Production 47(1), 377-387.

Teunter, R. H., Van der Lannb, E., \& Inderfurtha, K. (2000) How to set the holding cost rates in average cost inventory models with reverse logistics? Omega, 28(1), 409-415.

Recebido em: 21 dez. 2017 / Aprovado em: 02 abr. 2018

Para referenciar este texto

American Psychological Association (APA)

Hammes, G., Nilson, M., Silva, F. L. da., Rodriguez, C. M. T., \& Lezana, A. G. R. (2020). Mapeamento dos custos de logística reversa em uma indústria de embalagens. Exacta, 18(3), 668-685. https://doi.org/10.5585/ExactaEP.v18n3.8200. 